\title{
Descripción Anatómica de los Músculos del Miembro Pélvico de Puma (Puma concolor)
}

\author{
Anatomical Description of the Pelvic Limb Muscles of Puma (Puma Concolor)
}

\author{
César Caro-Munizaga* \& Ismael Concha-Albornoz*
}

CARO-MUNIZAGA, C. \& CONCHA-ALBORNOZ, I. Descripción anatómica de los músculos del miembro pélvico de puma (Puma concolor). Int. J. Morphol., 32(3):1085-1094, 2014.

RESUMEN: Los carnívoros son animales que ejercen fascinación sobre las personas. Históricamente, han formado parte de la cultura y mitología humana por millones de años. En la medicina, tanto la estructura como la función son inseparables como base y fundamento esta ciencia y arte y en conjunto son asignaturas básicas en la formación médica. El puma es uno de los grandes felinos de los que se conoce su ecología, reproducción y algunos aspectos de su nutrición. Sin embargo, los aspectos relacionados con su anatomía son muy generales. Es probable que por ser un animal protegido, que sólo podría ser obtenido y preservado para estudios sólo bajo condiciones especiales, se prefiera homologar su anatomía con la del gato doméstico por ejemplo. Se realizó una disección clásica de los miembros pélvicos de un espécimen de puma macho adulto, la que se llevó a cabo sobre distintas regiones topográficas de los miembros pélvicos de esta muestra. El estudio comparativo demostró mediante la descripción de las estructuras musculares y de sus anexos que el gato doméstico puede ser utilizado como un modelo anatómico para ser extrapolado en futuros estudios de estas estructuras en el puma, salvo algunas diferencias en ciertos puntos de origen, inserción y en el desarrollo de algunas estructuras musculares.

PALABRAS CLAVE: Veterinaria; Miología: Felinos; Puma.

\section{INTRODUCCIÓN}

Los carnívoros son animales que ejercen fascinación sobre las personas, siendo frecuentemente utilizados como modelos de fuerza y belleza en los medios publicitarios. Históricamente, han formado parte de la cultura y mitología humana por millones de años (Palacios, 2007).

El puma como carnívoro, representa uno de los grupos clave en el funcionamiento de ecosistemas naturales, ya que, mantiene el equilibrio entre las poblaciones naturales de presas y su ecosistema. Es además un posible indicador de presencia y abundancia de sus presas y del buen estado de conservación del ecosistema (Palacios; HernándezGuzmán et al., 2011).

El puma, junto con el jaguar, son los felinos depredadores más grandes de América. Su distribución original comprendía el Norte, Centro y Sur de América desde los cero hasta los $3000 \mathrm{~m}$ sobre el nivel del mar. Se encuentran amenazados principalmente por la pérdida y destrucción su de hábitat, la cacería indiscriminada de sus presas naturales y los conflictos con los humanos, por lo que han sido catalogados por la Unión Mundial para la Conservación de la Naturaleza (UICN, por sus siglas en inglés) como especies amenazadas (el jaguar como especie amenazada y el puma como de preocupación menor) (Isasi-Catalá \& Barreto, 2008).

El puma es uno de los grandes felinos de los que se conoce su ecología, reproducción y algunos aspectos de su nutrición. Sin embargo, los aspectos relacionados con su anatomía son muy generales. Es probable que por ser un animal protegido, que sólo podría ser obtenido y preservado para estudios sólo bajo condiciones especiales, se prefiera homologar su anatomía con la del gato doméstico por ejemplo (Concha et al., 2004).

El conocimiento general de la musculatura del miembro pélvico tiene numerosas aplicaciones en cirugía y medicina. Algunas de estas incluyen aproximaciones ortopédicas, diagnóstico de neuropatías, miopatías específicas y una comprensión fundamental de la conformación y de la locomoción (Shively, 1993). 


\section{MATERIAL Y MÉTODO}

Para el desarrollo de este trabajo se utilizó material biológico correspondiente a los miembros pélvicos de un ejemplar de puma, macho, adulto, aproximadamente de tres años edad, el que fue donado por el Servicio Agrícola y Ganadero (SAG) a la Unidad de Anatomía Veterinaria de la Universidad Santo Tomás, Santiago. Dicho ejemplar está conservado en solución fijadora-conservadora y cuenta con inyección vascular de látex y tinta china de colores azul (para distinguir vasos sanguíneos venosos) y rojo (para distinguir vasos sanguíneos arteriales). Se utilizó instrumental básico de disección (hojas de bisturí n ${ }^{\circ} 15,22$ y 24, mangos de bisturí nº y y , pinzas anatómica y quirúrgica, tijeras estándar recta roma-aguda, tijeras mayo y pinzas hemostáticas mosquito.

La metodología a seguir en este trabajo fue una disección clásica de los miembros pélvicos de la muestra en estudio. Esta disección se llevó a cabo sobre distintas regiones topográficas de los miembros pélvicos de la muestra, considerando las siguientes regiones: glútea, femoral, crural y pie, comenzando por la región más proximal (región glútea), siguiendo hacia distal y realizando disecciones desde superficial a profundo en cada región abordada.

Los diferentes planos de disección se documentaron fotográficamente con una cámara kódak easyshare ${ }^{\circledR}$ modelo C613 de 6,2 megapíxeles de resolución. La comparación de los músculos identificados se realizó en base a bibliografía referente a la anatomía del gato doméstico, contrastando lo encontrado en la disección con lo hallado en la literatura clásica.

\section{RESULTADOS Y DISCUSIÓN}

\section{Músculos glúteos}

Músculo glúteo superficial. Coincide con lo descrito para el gato doméstico; el músculo glúteo superficial fue el más superficial del grupo glúteo y a su vez el más caudal de este grupo muscular. Se halló caudal y superficial al músculo glúteo medio, craneal al músculo gluteofemoral y su extremo distal se encontró cubierto por el músculo bíceps femoral. Se observó este músculo conformando el contorno caudal de la región glútea.

Origen: fascia glútea.

Inserción: inmediatamente distal al trocánter mayor del fémur.

1086
Músculo glúteo medio. Este músculo fue el de mayor desarrollo en el grupo glúteo.

Origen: en cuanto a lo descrito con respecto al origen del músculo glúteo medio existen algunas diferencias. Sisson \& Grossman (1982) mencionan que el origen de este músculo es en la totalidad de la cara glútea del ilion, mientras que, König \& Liebich (2004) describen el origen del músculo glúteo medio en la cresta ilíaca y en la línea glútea. Según lo observado en la muestra anatómica diseccionada el origen del músculo glúteo medio es en las espinas ilíacas dorsales craneal y caudal. También Sisson \& Grossman afirman que algunas fibras musculares tienen su origen en la fascia glútea, lo que también fue observado en la disección.

Inserción: trocánter mayor del fémur.

Músculo glúteo profundo. Se observó como un músculo corto y fuerte, en forma de abanico, posicionado directamente sobre la articulación coxal y cubierto completamente por el músculo glúteo medio.

Sisson \& Grossman afirman que el músculo glúteo profundo se haya cubierto en su totalidad por los músculos glúteo medio y piriforme. Esto difiere de lo visto en la muestra anatómica en cuanto a que sólo el músculo glúteo medio cubre al músculo glúteo profundo, en tanto que el músculo piriforme lo cubre sólo en su mitad caudal.

Origen: Sisson \& Grossman y König \& Liebich describen que este músculo surge a lo largo del margen lateral del cuerpo de ilion y de la espina isquiática, lo que contrasta con lo observado en la disección, puesto que, en la muestra anatómica el músculo glúteo profundo tiene su origen en la cara glútea del ala y en el cuerpo del ilion.

Inserción: trocánter mayor del fémur.

Músculo piriforme. Se observó en la región de la incisura isquiática mayor, pasando por sobre el trocánter mayor del fémur. Se observó profundo al m. glúteo medio y al m. glúteo superficial.

Origen: se origina en el extremo caudal del sacro, por lateral.

Inserción: trocánter mayor del el fémur.

\section{Músculos profundos relacionados con la articulación coxal}

Músculo articular coxal. Se halló craneolateral a la articulación coxal, cruzando la superficie flexora de la articula- 
ción coxal, caudolateral al músculo recto femoral y profundo al $\mathrm{m}$. glúteo profundo. No se observaron grandes diferencias con lo descrito para el gato doméstico, excepto por el gran desarrollo que presentó este músculo.

Origen: en la superficie lateral del cuerpo del ilion.

Inserción: en el cuello femoral, entre los músculos vasto lateral e intermedio, junto a la cápsula articular coxal.

Músculo obturador interno. Las fibras de este músculo convergen hacia la incisura isquiática menor, para formar un tendón plano, fuerte y largo, el que recorre hacia lateral, dorsal a la incisura isquiática menor.

Origen: superficie dorsal del piso de la pelvis alrededor del agujero obturador.

Inserción: fosa trocantérica del fémur.

Músculo obturador externo. Se halló cubriendo ventralmente al agujero obturador ipsilateral. Las fibras de este músculo se dirigen hacia lateral. Su margen caudal se encontró profundo al cuadrado femoral, mientras que el margen craneal se observó profundo a los músculos aductores.

Origen: en la superficie ventral del pubis y del isquion.

Inserción: fosa trocantérica del fémur.

Músculos gemelos. Estos músculos se hallaron relacionados al tendón del m. obturador interno. Estos músculos fueron diferenciables por el tendón del músculo obturador interno, puesto que en el margen craneal de este último se encontró el m. gemelo craneal, mientras que en el margen caudal se halló el m. Gemelo caudal.

Origen: el m. gemelo craneal tiene su origen en la espina isquiática, mientras que, el $\mathrm{m}$. gemelo caudal se origina en el tubérculo isquiático, entre los músculos obturador interno y bíceps femoral.

Inserción: fosa trocantérica del fémur.

Músculo cuadrado femoral. Se distinguió como un músculo corto y grueso situado profundo al bíceps femoral, entre el $\mathrm{m}$. aductor en dirección medial y el m. gemelo caudal en sentido craneal.

Origen: superficie ventral del tubérculo isquiático.

Inserción: en la parte caudal del fémur, inmediatamente distal a la fosa trocantérica.

\section{Músculos de la región femoral caudal}

Músculo bíceps femoral. Correspondió al más largo y ancho de los músculos de la región femoral, comprendiendo la región glútea, la región femoral lateral y la región crural lateral. Se extendió distalmente dividiéndose en dos cabezas. La porción craneal es la de mayor superficie, se inserta tanto en la fascia lata como en la fascia crural por lateral, además de la contribución a la formación del tendón calcáneo común.

La porción caudal se distingue separada longitudinalmente de la porción principal del músculo bíceps femoral, la que se origina desde el tubérculo isquiático por un tendón definido y en ella se observan fibras musculares que se dirigen hacia distal.

Sisson \& Grossman describen que la mayor parte de las fibras del músculo bíceps femoral discurren en dirección craneodistal, lo que coincide con lo observado en la muestra anatómica, pero sólo en los dos tercios craneales de la porción craneal de este músculo, en tanto que en el tercio caudal de la porción craneal del m. bíceps femoral, es decir, en el área entre las porciones de este músculo, se observan las fibras musculares en una orientación más caudocraneal.

Origen: es descrito por Sisson \& Grossman en la tuberosidad isquiática en sus dos porciones (craneal y caudal), lo que contrasta con lo descrito por König \& Liebich que mencionan que este músculo consta de una porción craneal notoria que se origina en el sacro y de una porción caudal, más débil que nace en el isquion. Al observar el espécimen estudiado se concluye que el músculo bíceps femoral nace desde la tuberosidad isquiática en sus dos porciones, concordando con lo descrito por Sisson \& Grossman. Es importante señalar, además, que a diferencia del canino doméstico (Canis lupus familiaris) los felinos no presentan ligamento sacrotuberal, estructura que en caninos es un punto de origen del m. bíceps femoral en conjunto con el tubérculo isquiático (Evans \& DeLaHunta, 2002).

Inserción: fascia lata y fascia crural por lateral. También se distinguió participación en la formación del tendón calcáneo común.

Músculo gluteofemoral. El músculo gluteofemoral se encontró profundo al bíceps femoral en su tercio medio y en su tendón de inserción. Su tendón de origen se observó entre los músculos glúteo superficial por craneal y bíceps femoral, por caudal.

Cabe señalar que el músculo gluteofemoral corresponde al anteriormente llamado músculo caudofemoral o 
abductor crural craneal (International Commitee on Veterinary Gross Anatomical Nomenclature, 2005).

Origen: Sisson \& Grossman y Done et al. (1997) describen que el músculo caudofemoral se origina desde las primeras tres vértebras caudales a diferencia delo descrito por König $\&$ Liebich, donde se describe que este músculo se origina en las vértebras coccígeas segunda a cuarta. Según lo observado en la muestra en estudio el músculo gluteofemoral se originó desde las primeras tres vértebras coccígeas, siendo esto concordante con lo descrito por Sisson \& Grossman y Done et al.

Inserción: König \& Liebich describen la inserción de este músculo de forma aponeurótica en la fascia lata y lateralmente en la patela, mientras que Sisson \& Grossman y Done et al. describen que en el gato doméstico este músculo se inserta en la fascia craneal del bíceps femoral y una pequeña porción desciende distalmente hasta la patela. Lo observado en el espécimen en estudio entrega una información distinta a lo descrito por estos autores para el gato doméstico. Se observó la inserción de este músculo por medio de un tendón aponeurótico único en el cóndilo lateral del fémur y en el tendón de origen de la cabeza lateral del m. gemelo.

Músculo semitendinoso. Se observó muy desarrollado, dándole el contorno al margen caudal de la región femoral (Fig.1). Se distinguió la parte proximal de este músculo en la región femoral lateral y cerca de su origen se relaciona hacia lateral con el $\mathrm{m}$. bíceps femoral y hacia medial con el $\mathrm{m}$. semimembranoso. Se observó que este músculo se dirige hacia distal, caudal al bíceps femoral, para situarse medialmente a la fosa poplítea y los músculos caudales de la región crural. Cerca de su inserción se halló relacionado con la cabeza medial del $\mathrm{m}$. gastrocnemio y $\mathrm{m}$. gracilis.

Origen: ventral al tubérculo isquiático.

Inserción: es descrita por Sisson \& Grossman en el margen craneal de la tibia, a diferencia de lo descrito por König \& Liebich quien describe la inserción de este músculo en la cara medial del cuerpo de este mismo hueso (en ambos casos obviando su participación en la formación del tendón calcáneo común). Lo observado en la muestra anatómica denota que la inserción de este músculo coincide con lo descrito por Sisson \& Grossman, donde este último además agrega que el músculo semitendinoso se inserta medialmente en conjunto con los tendones terminales del $\mathrm{m}$. gracilis y $\mathrm{m}$. sartorio.

También Sisson \& Grossman describen que la porción media del músculo contiene una inserción tendinosa, información coincidió con lo observado en el espécimen diseccionado, con la diferencia de que esta inserción se ubicó en el tercio proximal del músculo. Se observó además la participación del $\mathrm{m}$. semitendinoso en la formación del tendón calcáneo común mediante un fuerte tendón aponeurótico que pasa medial al gastrocnemio.

Músculo semimembranoso (Fig.1). Se encontró en su mayor parte relacionado medialmente con el músculo gracilis. Se encontró con un desarrollo mayor en comparación al semitendinoso, pero con una longitud menor. Fue posible distinguir dos porciones adyacentes una a la otra y con dimensiones similares.

Origen: tubérculo isquiático, caudomedial al m. semitendinoso.

Inserción: lo descrito para la porción craneal por Sisson \& Grossman y König \& Liebich en cuanto a su inserción es que esta tiende a ser proximal a la porción caudal y, por tanto, se une al epicóndilo medial del fémur y al origen del m. gastrocnemio, lo que coincidió sólo en la inserción más proximal (epicóndilo medial del fémur), mientras que, lo descrito para la porción caudal es que esta se inserta en el cóndilo medial de la tibia, lo que también fue concordante con la muestra observada.

Músculo abductor crural caudal. Este músculo se encontró profundo al bíceps femoral, adherido a la superficie medial dela cabeza caudal de este (Fig. 1). Se observó como una cinta muscular angosta.

Origen: Done et al. describen que el músculo abductor crural caudal del felino se origina en las vértebras coccígeas 2 y 3 , lo que fue distinto a lo observado en la muestra anatómica en estudio, donde el músculo abductor crural caudal tuvo su origen en la fascia glútea, en conjunto con el músculo glúteo superficial.

Inserción: fascia crural, en conjunto con las fibras más caudales del músculo bíceps femoral.

\section{Músculos de la región femoral medial.}

Músculo sartorio. Se observó como el más craneal de la región femoral medial, de tal manera que le otorgó el contorno craneal a la región femoral (Fig. 1). Al igual que en el gato doméstico este músculo se halló sin divisiones y se formó de una porción que se extendió desde la tuberosidad coxal hasta la superficie medial de la articulación genual. Su porción más proximal se encontró en la región femoral por craneal y a medida que se dirigió hacia distal se posicionó en dirección medial hasta llegar a la región de la articulación genual. Se denotó además un gran desarrollo en este 


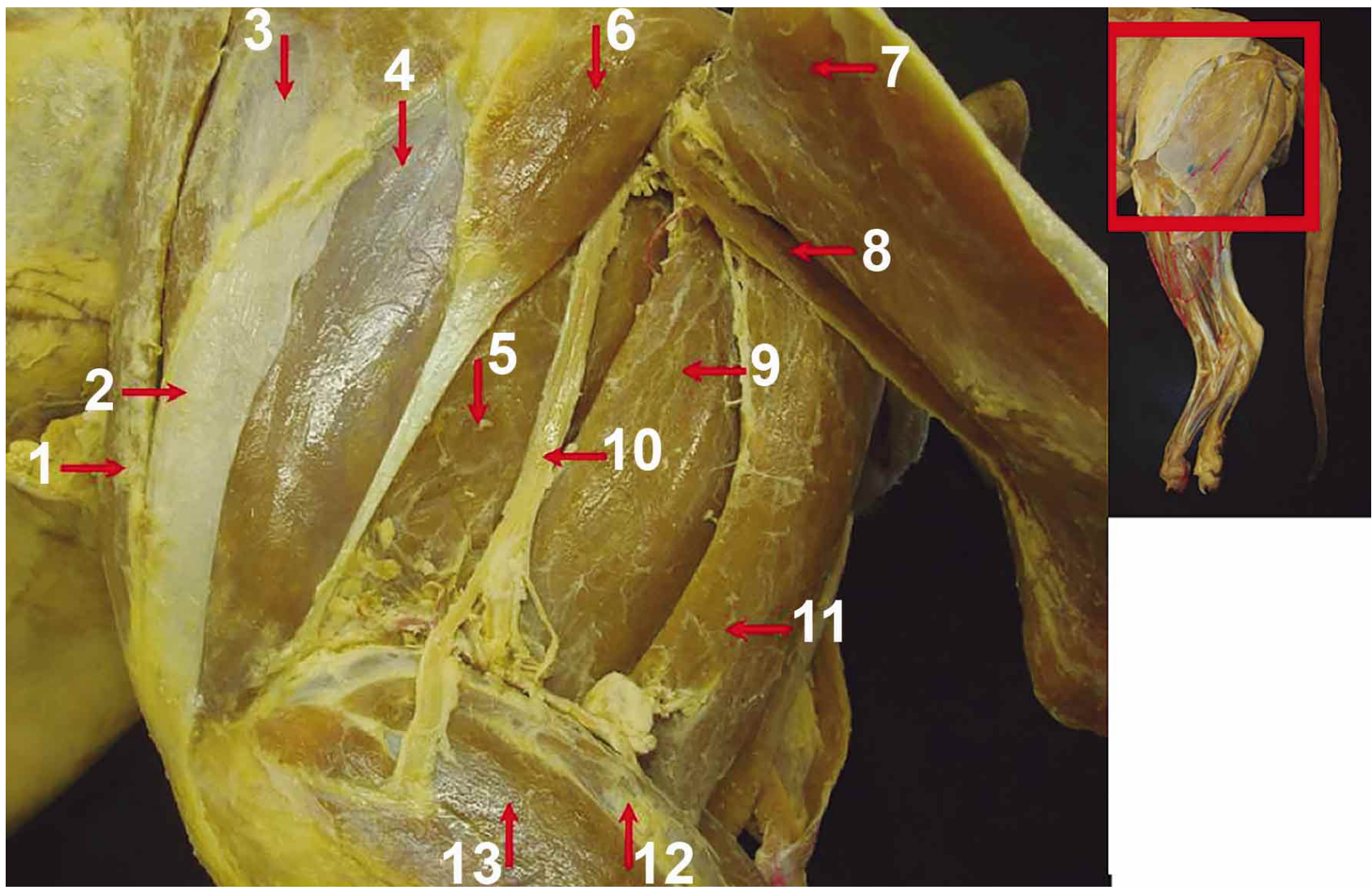

Fig- 1. Músculos de la región femoral lateral izquierda de puma, vista lateral. Se han rechazado hacia caudal los músculos bíceps femoral y abductor crural caudal. 1. Sartorio; 2. Fascia lata seccionada; 3. Tensor de la fascia lata; 4. Vasto lateral; 5. Aductor mayor y breve; 6. Gluteofemoral; 7. Bíceps femoral rechazado hacia caudal; 8. Abductor crural caudal rechazado hacia caudal; 9. Semimembranoso; 10. Nervio isquiático; 11. Semitendinoso; 12. Flexor digital superficial; 13. Gastrocnemio, cabeza lateral.

músculo, el que se observó como una lámina muscular, de una conformación similar al músculo gracilis. Además se observó un tendón de inserción aponeurótico amplio que abarcó el extremo distal del tendón del cuádriceps femoral, la patela y el tendón patelar, continuándose hacia distal con una inserción conformada por fascia en la tuberosidad tibial y en la fascia crural.

Origen: espina ilíaca craneoventral y fascia toracolumbar.

Inserción: región de la articulación genual por medial mediante aponeurosis y además en la fascia crural.

Músculo pectíneo. Se observó como un músculo fusiforme ubicado craneal al aductor y caudal al vasto medial. En su recorrido se dirigió hacia distal a través de un surco formado entre el vasto medial y el aductor. A diferencia de lo descrito en para el gato doméstico no se observó un largo tendón aponeurótico de inserción.

Origen: tendón prepúbico y pecten pubis.
Inserción: margen medial de la cara áspera del fémur, mediante aponeurosis.

Músculo gracilis. Se observó como una lámina muscular amplia y delgada en la cara medial de la región femoral. Este músculo fue el más superficial de esta región. Además se observó su participación en la formación del tendón calcáneo común.

Origen: se observó un origen aponeurótico en el tendón sinfisiario el que era compartido con el gracilis contralateral, el que a su vez se observó inserto en la superficie ventral de la sínfisis isquiopubiana.

Inserción: a lo largo de todo el margen craneal de la tibia a través de un tendón plano y fuerte que se encontró profundo al sartorio.

Músculo aductor. Este músculo abarcó la parte caudal de los músculos profundos de la región femoral medial y se observaron sus dos porciones (aductor mayor y breve y 
aductor largo) fusionadas, observándose una línea débil entre ambos músculos.

El aductor se halló entre el pectíneo y el semimembranoso. Se observaron sus fibras musculares en dirección dorso ventral caudo craneal (Fig.1).

Origen: sínfisis isquiopubiana, el tendón sinfisiario y el tubérculo público.

Inserción: distal a la fosa trocantérica y en la cara áspera del fémur.

\section{Músculos craneales de la región femoral}

Músculo tensor de la fascia lata. Se encontró en la región femoral craneal, se observó de una forma piramidal con caras lateral, craneal y caudal, una base hacia proximal y un vértice hacia distal (Fig. 1): la base, de orientación proximal, presentó una fosa excavada o una impresión para el m. glúteo medio, con el que se relaciona hacia proximal. La cara craneal tomó relación con el m. sartorio, la cara caudal se halló relacionada con el m. cuádriceps femoral y la cara lateral se encontró cubierta por la fascia lata y el tegumento de la región femoral.

Origen: espina iliaca craneoventral, en la porción lateral del ilion y en el tendón de inserción del músculo glúteo medio. Inserción: se observó la inserción del m. tensor de la fascia lata mediante una aponeurosis amplia (Fig.1), que se continuó hacia caudal con el margen craneal del m. bíceps femoral. Se distinguió además en esta aponeurosis una inserción distal en el tendón patelar, en conjunto con el m. sartorio. Finalmente se observó una profundización hacia medial de la cara craneal del $\mathrm{m}$. tensor de la fascia lata, que se relacionó con el vasto medial.

Músculo cuádriceps femoral. Fue el músculo más desarrollado de los músculos craneales de la región femoral y del miembro pélvico en su totalidad. Este músculo se encontró rodeando al fémur en sus superficies medial, craneal y lateral. Se observaron cuatro cabezas: recto femoral, vasto lateral (Fig. 1), vasto medial y vasto intermedio, las que convergieron en un tendón común sobre la patela, formando el tendón patelar, el que se insertó en la tuberosidad tibial. El recto femoral fue la cabeza de mayor desarrollo y la más craneal.

Origen: los músculos vastos se originaron desde el fémur en su extremo proximal, mientras que el origen del recto femoral se halló, craneal al acetábulo.

Inserción: las cuatro cabezas convergieron en un tendón común superficial a la patela, formando el tendón patelar, el que se insertó en la tuberosidad tibial.

Músculo articular genual. Este es un músculo pequeño y corto que se encontró en el extremo distal de la diáfisis del fémur, entre los músculos recto femoral y vasto lateral.

Origen: en el fémur inmediatamente proximal a la tróclea femoral.

Inserción: receso supratroclear y cápsula articular, inmediatamente proximal a la patela.

\section{Músculos craneolaterales de la región crural}

Músculo tibial craneal. Fue el músculo más craneal, medial y superficial de este grupo (Fig. 2). Su margen medial se halló en contacto con la tibia. Se observó los tendones de origen del $\mathrm{m}$. tibial craneal y del $\mathrm{m}$. fibular largo formar un retináculo para el tendón de origen del $\mathrm{m}$. extensor digital largo a nivel del surco extensor de la tibia.

Se observó la formación del tendón del m. tibial craneal a nivel del tercio distal de la tibia, el que se encontró sujeto por el retináculo extensor proximal en conjunto con el músculo extensor digital largo, dorsal al tarso.

Origen: König \& Liebich mencionan que este músculo tiene su origen en el cóndilo lateral de la tibia y en la epífisis proximal de la fíbula. Las observaciones llevadas a cabo en la muestra anatómica demostraron concordancia con lo descrito por König \& Liebich.

Inserción: según Sisson \& Grossman y König \& Liebich el tendón del tibial craneal se inserta en el lado medial de la región metatarsal en la superficie plantar de las bases del rudimento del primer metatarsiano y del segundo metatarsiano, lo que coincidió, en parte, con lo observado en el espécimen en estudio, puesto que, se encontró sólo un sitio de inserción, que correspondió a la superficie plantar de la epífisis proximal del segundo metatarsiano, puesto que, el puma presentó en el miembro pélvico sólo los dedos II, III, IV y V.

\section{Músculo extensor digital largo I. No se observó en el espécimen en estudio.}

Músculo fibular largo. Se encontró inmediatamente caudal al extensor digital largo (Fig. 2). Se halló cubierto directamente por la fascia crural. Se observó un largo y estrecho tendón que se encontró caudal al maléolo lateral. 
Origen: cóndilo lateral de la tibia, en el ligamento colateral lateral y el extremo proximal de la fíbula.

Inserción: según Sisson \& Grossman el tendón del músculo fibular largo se inserta en el cuarto hueso tarsiano y en los extremos proximales de los metatarsianos, a diferencia de lo descrito por König \& Liebich quienes mencionan que este músculo se inserta en el primer hueso tarsiano y en el metatarso II. Lo observado en la muestra anatómica en estudio mostró que la inserción de este músculo se encuentra en a cara plantar de la epífisis proximal del metatarso V.

Músculo fibular corto. Se encontró profundo al fibular largo y proximal al maléolo lateral (Fig. 2). En el extremo proximal su tendón recorrió caudal al tendón del músculo extensor digital lateral, mientras que, en su extremo más distal este se halló caudal al tendón del fibular largo. Su tendón de inserción pasa en el surco caudal del maléolo lateral, dirigiéndose distalmente y en profundidad al tendón del m. fibular largo.

Origen: desde los tres cuartos proximales de la tibia y fíbula.

\section{Inserción: extremo proximal del metatarso V.}

Músculo extensor digital largo. Se distinguió su origen en el cóndilo lateral de la tibia, posteriormente este músculo pasó por el surco extensor del mismo hueso. Se observó su cuerpo muscular de un aspecto fusiforme, ubicado entre los músculos tibial craneal y fibular largo (Fig. 2). Además se observó sujeto por el retináculo extensor proximal, junto con el tendón del m. tibial craneal, posteriormente pasó por la superficie dorsal del tarso y se encontró sujeto al retináculo extensor distal. Finalmente se dividió en cuatro tendones, los que se dirigieron hacia el proceso extensor de la falange distal de los dedos II, III, IV y V.

Origen: cóndilo lateral de la tibia.

Inserción: proceso extensor de las falanges distales de los dedos II, III, IV y V.

Músculo extensor digital lateral. Se encontró cerca de su origen profundo al músculo fibular largo (Fig. 2), se convirtió prontamente después de su origen en un tendón que descendió entre los músculos fibulares largo y corto. El tendón del músculo extensor digital lateral sufrió un cambio de dirección a nivel de la articulación tarsal y pasó profundo al tendón del músculo fibular largo. El tendón del músculo extensor digital lateral se encontró incluido en una vaina sinovial en conjunto con el tendón del músculo fibular corto, en el surco caudal del maléolo lateral. Además se distinguió una fusión de gran parte de la masa de estos dos músculos.
Origen: Sisson \& Grossman mencionan que el músculo extensor digital lateral se origina desde la mitad distal del tercio proximal de la fíbula, mientras que, König \& Liebich describen el origen de este músculo desde la fíbula y desde el ligamento colateral lateral de la articulación genual. Las observaciones demostraron que este músculo se origina desde la fíbula y su masa muscular se encuentra adherida la del músculo fibular corto.

Inserción: cerca de su inserción se unió al tendón del músculo extensor digital largo, el que se insertó en el proceso extensor de la falange distal del quinto dedo.

\section{Músculos caudales de la región crural}

Músculo gastrocnemio. Fue el músculo más superficial de los músculos caudales de la región crural y fue el que le otorga el contorno caudal a la región crural (Fig. 2). Se observaron dos cabezas, las que envolvieron de manera superficial al músculo flexor digital superficial. En cada tendón de origen se observó un hueso sesamoideo articulando con la cara caudodorsal del cóndilo femoral ipsilateral.

Origen: las cabezas medial y lateral se originaron desde las tuberosidades supracondilares medial y lateral del fémur respectivamente.

Inserción: hacia distal las porciones del gastrocnemio se unieron para formar un tendón de inserción que terminó en la tuberosidad calcánea, este tendón fue el que presentó una mayor participación en la formación del tendón calcáneo común.

Músculo sóleo. Se encontró muy bien desarrollado y se observó lateral y craneal al vientre lateral del gastrocnemio y caudal al músculo fibular largo (Fig. 2).

Origen: cóndilo lateral de la tibia.

Inserción: tuberosidad calcánea, participando también en la formación del tendón calcáneo común.

Músculo poplíteo. Se encontró en la en la cara caudal de la tibia. Cerca de su origen se halló profundo al ligamento colateral lateral de la articulación genual. Se observó un largo tendón de origen, luego este músculo se dirigió hasta el tercio proximal de la tibia, superficial al flexor digital profundo. En surecorrido pasó a través de la fosa poplítea en la cara caudal de la tibia, donde se observó un hueso sesamoideo.

Origen: cóndilo lateral del fémur 
Inserción: Sisson \& Grossman describen la inserción del músculo poplíteo en la cara medial del tercio proximal de la tibia, mientras que, König \& Liebich describen la inserción de este músculo en la cara caudal del tercio proximal de la tibia. Lo observado en la muestra anatómica concordó con lo descrito por Sisson \& Grossman.

Músculo flexor digital superficial. Se encontró superficial a los músculos flexor digital profundo y poplíteo y profundo a los músculos sóleo y gastrocnemio.

Origen: Sisson \& Grossman mencionan que el músculo flexor digital superficial se origina en la fosa poplítea de la tibia. König \& Liebich mencionan solamente a la tuberosidad supracondilar lateral del fémur como sitio de origen del músculo flexor digital superficial. Según lo observado el músculo flexor digital superficial se originó en la tuberosidad supracondilar del fémur en conjunto con la cabeza lateral del músculo gastrocnemio. También se observó, a nivel de la cara plantar de las falanges proximales de los dedos II, III, IV y V, la formación de la mánica flexora por parte del tendón del músculo flexor digital superficial, la que es perforada por el tendón del músculo flexor digital profundo.

Inserción: tuberosidad calcánea, lugar donde formó parte del tendón calcáneo común. Este tendón de inserción luego se continuó hacia distal en la superficie plantar de la región metatarsal en donde a partir del tendón de inserción originó cuatro tendones que se insertaron en la cara plantar de la epífisis proximal de la falange media.

Músculo flexor digital profundo. Fue el músculo más profundo de la región crural caudal (Fig. 2). Se le distinguieron tres porciones: flexor digital lateral, flexor digital medial y tibial caudal. En cuanto al desarrollo de estas porciones se puede mencionar que el de mayor tamaño fue el flexor digital lateral y la de menor desarrollo fue el flexor digital medial. El tendón del músculo flexor digital lateral pasó por el sustentáculo talar (estructura correspondiente al hueso calcáneo), mientras que, el tendón del flexor digital medial pasó por la cara plantar del talo. Distalmente ambos tendones se unieron a nivel de la cara plantar del tarso para formar el tendón del flexor digital profundo, que recorrió la superficie plantar de la región del pie, donde se dividió en cuatro tendones (uno para cada dedo), luego a nivel de la superficie plantar de la falange proximal perforó al tendón del flexor digital superficial para finalmente insertarse en el proceso flexor de la falange distal de los dedos II, III, IV y V. El músculo tibial caudal se observó en la cara plantar del tarso hacia medial, pasando plantar al ligamento colateral medial del tarso e insertándose de manera independiente al tendón principal del flexor digital profundo.
Origen: desde la superficie caudal de los cóndilos tibiales y desde la fosa poplítea en cara caudal de la tibia.

Inserción: los tendones de las porciones medial y lateral se insertaron en los procesos flexores de las falanges distales de los dedos II, III, IV y V. El tendón de la porción tibial caudal se insertó en la cara plantar de la base del metatarso II.

\section{Músculos de la región del pie}

Músculo extensor digital corto. Se ubicó en la superficie dorsal de la región del pie. Desde su sitio de origen emergieron tres tendones: uno lateral, uno medio y otro medial.

Origen: fila distal de los huesos tarsianos.

Inserción: el tendón medio se dividió en dos para insertarse en la superficie plantar de los tendones del músculo extensor digital largo que se dirigían hacia los dedos III y IV, mientras que, los tendones medial y lateral se insertaron en los tendones del extensor digital largo II y $\mathrm{V}$ respectivamente.

\section{Músculo cuadrado plantar. No se halló en el especimen en estudio.}

Músculo cuadrado interflexor. No se halló en el espécimen en estudio.

Músculos lumbricales. Se observaron como estructuras musculares entre los tendones de los músculos flexores digitales superficial y profundo.

Origen: cara plantar del tendón del músculo flexor digital profundo, a nivel de la división de su este tendón.

Inserción: cara dorsal de los tendones del flexor digital superficial. Sisson \& Grossman y König \& Liebich describen la inserción de los músculos interflexores a nivel de la base de la falange proximal de los dedos II, III y V. En el espécimen en estudio se encuentra la inserción de los interflexores a nivel de la falange proximal de los dedos III, IV y V.

Músculo flexor digital corto. Se encontró como una delgada banda muscular en la cara lateroplantar de la región metatarsal.

Origen: según Sisson \& Grossman y König \& Liebich el músculo flexor digital corto se origina en el tendón del flexor digital superficial a la altura del tarso. Según lo observado en el espécimen en estudio a este lugar de origen se le agregó la cara lateral del metatarso $\mathrm{V}$. 

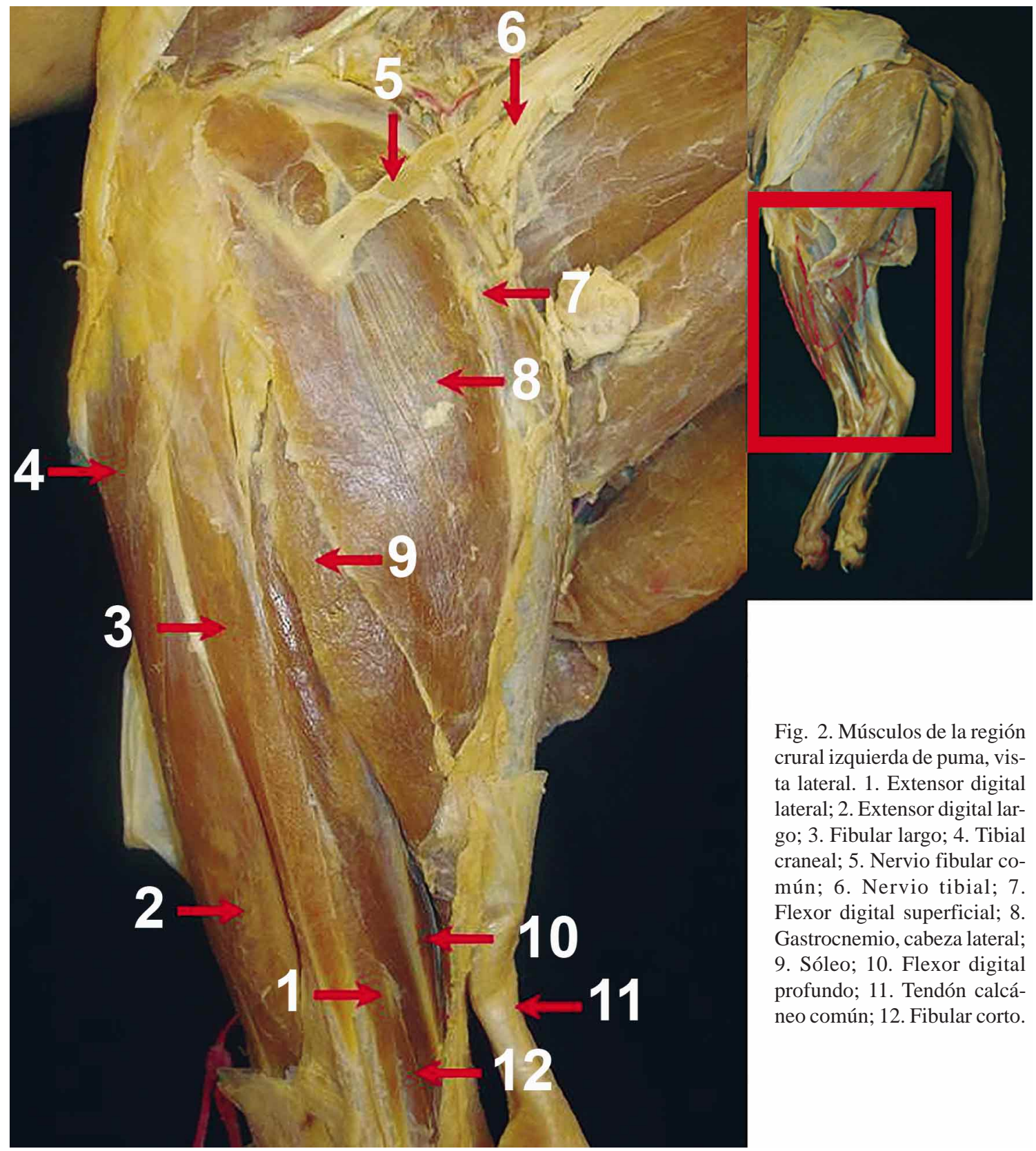

Fig. 2. Músculos de la región crural izquierda de puma, vista lateral. 1. Extensor digital lateral; 2. Extensor digital largo; 3. Fibular largo; 4. Tibial craneal; 5. Nervio fibular común; 6. Nervio tibial; 7. Flexor digital superficial; 8. Gastrocnemio, cabeza lateral; 9. Sóleo; 10. Flexor digital profundo; 11. Tendón calcáneo común; 12. Fibular corto.

Inserción: tendón del músculo flexor digital profundo a nivel de la articulación metatarsofalángica.

Músculos interóseos. Se observan a nivel de la superficie plantar de los metatarsos.

Origen: cara plantar de la base de los huesos metatarsianos,

Inserción: cara abaxial de los huesos sesamoideos metatarsofalángicos y márgenes medial y lateral de los tendones del extensor digital largo a nivel de la cara dor- sal de la falange proximal de los dedos II, III, IV y V.

Músculo abductor del dedo V. Se observó como una banda muscular superficial al cuadrado plantar. Sus fibras musculares se dirigieron hacia distal.

Origen: superficie lateral de la tuberosidad calcánea.

Inserción: en la base del metatarso V. Además se distinguió un tendón de inserción adicional que se dirigió hasta el maléolo lateral. 
CARO-MUNIZAGA, C. \& CONCHA-ALBORNOZ, I. Anatomical description of the pelvic limb muscles of puma (Puma concolor). Int. J. Morphol., 32(3):1085-1094, 2014.

SUMMARY: Carnivores are animals fascination exerted on people. Historically, they have been part of human culture and mythology for millions of years. In medicine, the structure and function are inseparable as the basis and foundation this science and art together are basic subjects in medical training. The cougar is one of the big cats are known for their ecology, reproduction and aspects of nutrition. However, aspects of their anatomy are very general. Is likely to be a protected animal, which could only be obtained and preserved for study only under special conditions, is preferred to standardize their anatomy such domestic cat. Was conducted classic dissection of the lower limbs of a specimen of adult male cougar, which took place on different topographic regions of the lower limbs of this sample. The comparative study demostrated by describing the muscular structures and its annexes that domestic cats can be used as an anatomical model to be extrapolated in future studies of these structures in the puma, except for some differences on certain points of origin and insertion in the development of some muscular structures.

KEY WORDS: Veterinary; Myology; Felines; Puma.

\section{REFERENCIAS BIBLIOGRÁFICAS}

Concha, I.; Adaro, L.; Borroni, C. \& Altamirano, C. Anatomical considerations over the intrinsic musculature of thoracic limb of puma (Puma concolor). Int. J. Morphol., 22(2):121-5, 2004.

Done, S.; Goody, P.; Evans, S. \& Stickland, N. Atlas en color de Anatomía Veterinaria. Madrid, Harcourt Brace, 1997.

Evans, H. \& DeLaHunta, A. Disección del perro. 4a ed. México D.F., McGraw Hill Interamericana, 2002. p.72.

Hernández-Guzmán, A; Payán, E. \& Monroy-Vilchis, O. Food habits of Puma concolor (Carnivora: Felidae) in the Parque Nacional Natural Purace, Colombia. Rev. Biol. Trop., 59(3):1285-94, 2011.

International Committee on Veterinary Gross Anatomical Nomenclature (I.C.V.G.A.N.). Nómina Anatómica Veterinaria. 5 a ed. Knoxville, Editorial Committee Hannover, Columbia, Gent, Sapporo, 2012. p.50.

Isasi-Catalá, E. \& Barreto, G. R. Identification of individual jaguars (Panthera onca) and pumas (Puma concolor) based on footprint morphometry (Carnivora: Felidae). Rev. Biol. Trop., 56(4):1893-904, 2008.

König, H. E. \& Liebich, H. G. Anatomía de los animales domésticos. Buenos Aires, Médica Panamericana, 2004. pp.236-64.

Palacios, R. Guía de campo para la identificación de carnívoros cordilleranos. Córdoba, Wildlife Conservation Network, 2007. Disponible en: http://www.gatoandino.org/archivos/ ManualIDcarnivorosRP-AGA.pdf

Shively, M. J. Anatomía Veterinaria básica, comparativa y clínica. México D.F., El Manual Moderno, 1993.

Sisson, S. \& Grossman, J. Anatomía de los animales domésticos. $5^{\mathrm{a}}$ ed. México D.F., Salvat Editores, 1982. pp.1678-87.
Dirección para Correspondencia:

Dr. César Caro Munizaga. DVM.

Unidad de Anatomía Veterinaria

Universidad Santo Tomás

Santiago

CHILE

Email: ccaromunizaga@live.cl

Recibido : 16-05-2014

Aceptado: 28-08-2014 